\title{
Molecular Characterization of Staphylococcus epidermidis Isolates Recovered from Patients Admitted to a Referral Hospital in Iran
}

\author{
Amirmorteza Ebrahimzadeh Namvar, ${ }^{1}$ Seyed Asghar Havaei, ${ }^{2,}{ }^{*}$ and Masoumeh Douraghi, ${ }^{3,4, *}$ \\ ${ }^{1}$ Department of Microbiology, Faculty of Medicine, Babol University of Medical Sciences, Babol, IR Iran \\ ${ }^{2}$ Department of Microbiology, School of Medicine, Isfahan University of Medical Sciences, Isfahan, IR Iran \\ ${ }^{3}$ Division of Microbiology, Department of Pathobiology, School of Public Health, Tehran University of Medical Sciences, Tehran, IR Iran \\ ${ }^{4}$ Food Microbiology Research Center, Tehran University of Medical Sciences, Tehran, IR Iran \\ "Corresponding authors: Masoumeh Douraghi, Ph.D., Division of Microbiology, Department of Pathobiology, School of Public Health, Tehran University of Medical Sciences, PO \\ Box: 14155-6446, Tehran, IR Iran. Tel: +98-2142933152, Fax: +98-2188954913, E-mail: mdouraghi@tums.ac.ir; Seyed Asghar Havaei, Ph.D., Department of Microbiology, School of \\ Medicine, Isfahan University of Medical Sciences, Isfahan, IR Iran. E-mail: Havaei@med.mui.ac.ir
}

Received 2016 April 22; Revised 2016 December 21; Accepted 2017 January 01.

\begin{abstract}
Background: Coagulase-negative Staphylococci (CoNS), especially Staphylococcus epidermidis, are considered as commensal bacteria of human skin and oral-nasal mucosa. Because of having many various virulence factors as well as the emergence of the multidrug-resistant (MDR) strains, this microorganism is regarded as a major cause of hospital-acquired bacteremia and invasive nosocomial infections.

Objectives: Due to the significance of S. epidermidisassociated infections in different health care units, the aim of this study was to determine the molecular epidemiology of S. epidermidis in true infection-associated isolates.

Methods: In our cross sectional study, a total of 183 S. epidermidis strains was collected during 8 months. Only 40 strains, which were identified as "true" infection-associated strains, were assessed via antibiotic susceptibility testing and pulsed field gel electrophoresis (PFGE) typing. Finally, strains with specific assigned pulsotypes were also analyzed by multilocus sequence typing (MLST).

Results: Specimens were most commonly obtained from the bloodstream, wound, and catheter. More than half (75\%) of the tested strains were found to be resistant to cefoxitin and ciprofloxacin (60\%), while $45 \%$ of strains showed resistance to tetracycline. Using PFGE, 3 clones (A, B and C) were identified. According to MLST, the frequency of ST2 and ST5 was more prominent than the other STs. Conclusions: In accordance with the life cycle of S. epidermidis, molecular characterization of invasive isolates is essential for controlling the epidemic strains in health care units.
\end{abstract}

Keywords: Nosocomial Infections, Multidrug-Resistance (MDR), Pulse Field Gel Electrophoresis (PFGE), Multilocus Sequence Typing (MLST), Staphylococcus epidermidis

\section{Background}

Coagulase-negative Staphylococci (CoNS), and especially Staphylococcus epidermidis, are considered to be commensal bacteria of human skin and oral-nasal mucosa (1). However, this microorganism is implicated in several opportunistic infections such as eye keratitis and endophthalmitis of contaminated contact lenses (2), urinary catheters (3), prosthetic joints, and bloodstream infections particularly in neonatal intensive care units (NICUs) (4). In addition, a wide variety of infections related to foreign bodies such as medical implant devices, peripheral or central intravenous catheters (CVCs), and indwelling catheters are also attributed to S. epidermidis (5). On the other hand, many virulence factors including elastase, lipase, esterase, delta toxin, and biofilm (polysaccharide intercellular adhesion) have been recognized in S. epidermidis (6).

S. epidermidis is known as a major cause of hospital- acquired bacteremia and invasive nosocomial infections $(7,8)$. Recently, the resistance of S. epidermidis to antimicrobial agents, particularly to methicillin, has been reported in various hospitals around the world (9). The emergence of methicillin-resistant S. epidermidis (MRSE) and also multidrug-resistant (MDR) strains are demonstrated worldwide (10). Moreover, the existence of a variety of adhesion factors and the ability for aggregation as well as biofilm formation are the main reasons for bacterial survival and circulation in the hospital setting.

The MRSE and also MDR strains are regarded as part of the repertoire of antimicrobial resistance genes that can be transferred to other staphylococci species $(11,12)$. Persistence of multidrug-resistant clones of S. epidermidis in health care units may lead to true infections in high-risk patients. Hence, the assessment of the route of infection and also discrimination between true infection and contamination is of prime importance. 


\section{Objectives}

The aim of this study was to determine the molecular epidemiology of S. epidermidis true infection-associated isolates recovered from admitted patients in a referral hospital of Isfahan..

\section{Methods}

\subsection{Setting}

This study was conducted at the Al-Zahra hospital; a main tertiary referral hospital affiliated with Isfahan University of Medical Sciences with 950 beds. This hospital provides tertiary services in different wards, including an intensive care unit (ICU) and nephrology, neonatal, oncology, urology, and neurology wards. The emergency unit of this hospital is the main referral center for patients from different parts of Isfahan and the neighboring provinces. Also, the Department of Pediatrics provides a variety of services for neonates and infants. According to the assessment of the ministry of health in 2010 - 2011, this center achieved the first rank among Iranian hospitals.

During the 8-month study period, several patients were screened for S. epidermidis infection/colonization. Demographic data and history of each case such as age, gender, ward of admission, site, and date of isolation were recorded. All S. epidermidis isolates were classified as either contaminated or true infections with following criteria: microscopic observation of pus cells (WBCs) in the specimens by a trained medical technologist in the hospital laboratory, a threefold isolation of bacteria, and an accepted colony count. Since the surveillance definition of the CDC states that bloodstream infections should be confirmed, necessary requirements such as patients with fever, hypothermia or hypotension were also taken into account (13).

\subsection{Identification of Isolates}

S. epidermidis isolates were identified using the following tests: colony morphology, Gram staining, catalase, coagulase test with rabbit plasma, DNase activity, mannitol fermentation, acid from trehalose, polymyxin B resistance, and other specific microbiological standard tests. The strains were stored in Trypticase soy broth (TSB) with $10 \%$ sterile glycerol at $-20^{\circ} \mathrm{C}$.

\subsection{Antibiotic Susceptibility Testing}

An antibiotic susceptibility test was performed using Muller Hinton Agar (MHA) by disk diffusion method with ten antibiotic discs (Mast, Merseyside, England) including: ciprofloxacin $(5 \mu \mathrm{g})$, gentamicin $(10 \mu \mathrm{g})$, clindamycin (2 $\mu \mathrm{g})$, cefoxitin $(30 \mu \mathrm{g})$, oxacillin $(1 \mu \mathrm{g})$, tetracycline (30 $\mu \mathrm{g})$, levofloxacin $(5 \mu \mathrm{g})$, trimethoprim $(1.25 \mu \mathrm{g})+$ sulfamethoxazole $(23.75 \mu \mathrm{g})$, and rifampin $(5 \mu \mathrm{g})$ according to the CLSI (2013) guidelines (14). Isolates with an intermediate level of susceptibility were classified as non-susceptible. We defined the S. epidermidis isolates as MDR strains by observing the resistance to at least 3 antibiotics of different classes.

\subsection{Pulsed Field Gel Electrophoresis (PFGE)}

Forty isolates, which were associated with true infections, were characterized by PFGE. PFGE was conducted as described by Chung et al. (15). In brief, one colony from each isolate was inoculated into a $5 \mathrm{~mL}$ Brain Heart Infusion Broth (BHI) and incubated for 24 hours at $35-37^{\circ} \mathrm{C}$. The optical density of each sample test reached $1.2-1.8$ at $600 \mathrm{~nm}$. Each plug was prepared by adding $120 \mu \mathrm{L}$ of bacterial suspension, $400 \mu \mathrm{L}$ EC buffer (10 mM EDTA, $1 \mathrm{M} \mathrm{NaCl}$, 6 mM Tris-HCl (pH 7.6), 0.2\% Deoxycholate, 0.5\% Sarkosyl and 0.5\% Brij-58), $20 \mu$ L Lysostaphin (1 mg/mL)(Sigma,USA) and $450 \mu \mathrm{L} 2 \%$ Seakem Gold agarose (FMC Bio-Products, Rock land, ME). By adding $1800 \mu \mathrm{L}$ EC buffer with $200 \mu \mathrm{L}$ lysozyme $(20 \mathrm{mg} / \mathrm{mL}$ ) (Sigma, USA), plugs were incubated in $37^{\circ} \mathrm{C}$ for 5 hours, then washed with $1 \times$ TE buffer $(10 \mathrm{mM}$ Tris-HCl, 1 mM EDTA). Thereafter, $1800 \mu \mathrm{L}$ ES buffer (0.4M EDTA, $1 \%$ Sarkosyl) plus $70 \mu \mathrm{L}$ of $50 \mathrm{mg} / \mathrm{mL}$ proteinase $\mathrm{K}$ (Sigma, USA) were added and incubated at $55^{\circ} \mathrm{C}$ overnight. The restriction digestion step was done by adding $30 \mathrm{U}$ SmaI (Takara, Japan) for an incubation of 16 - 20 hours at $30^{\circ} \mathrm{C}$ after washing the plug slices with TE buffer.

PFGE was performed with $1 \%$ agarose gel in a contourclamped homogeneous electric field (CHEF-DRIII) (Bio-Rad USA) apparatus for 22 hours overnight at $12^{\circ} \mathrm{C}$, with a constant voltage of $6 \mathrm{~V} / \mathrm{cm}$ and pulses ramped from 5 to 50 seconds at an angle of 120 degrees. XbaI (Takara, Japan) digested genomic DNA of the Salmonella enterica serovar Braenderup strain H9812, which was used as a molecular weight marker. Gels were photographed after staining in $0.5 \mu \mathrm{g} / \mathrm{mL}$ ethidium bromide. Finally, PFGE profile were analyzed with GelCompar II version 4 (Applied Maths, Sint-Martens-Latem, Belgium) with a $90 \%$ similarity cut-off point and clustered using the Dice's coefficient, then represented by unweighted pair grouping by mathematical averaging (UPGMA). The band pattern of each strain was also visually confirmed according to the principles stated by Tenover et al. (16).

\subsection{Multilocus Sequence Typing (MLST)}

Nine isolates of distinct pulsotypes were evaluated by MLST. MLST was carried out though comparing the nucleotide sequences of 7 housekeeping genes, consisting of carbamate kinase $(\operatorname{arcC})$, shikimate dehydrogenase (aroE), ABC transporter (gtr), DNA mismatch repair 
protein (mutS), pyrimidine operon regulatory protein (pyrR), triosephosphate isomerase (tpiA,) and acetyl-CoA acetyltransferase (yqiL), which was previously described by Thomas et al. (17). The alleles, allelic profiles, and the sequence type (STS) of each of the 7 housekeeping loci were obtained from the MLST database (http://www.mlst.net).

\subsection{Data Analysis}

All statistical analyses were performed with SPSS version 21 (SPSS Inc., Chicago, IL). Each PFGE type was considered as a pulsotype and the combination of allelic types was defined as sequence type (ST).

\section{Results}

In our cross sectional study among 183 recovered $S$. epidermidis isolates from different patients, 40 isolates were recognized as true infection-associated strains. The most commonly collected specimen was from the bloodstream (35\%) as well as from wounds (35\%), followed by catheter related infections (10\%), and other sites (20\%) including shunt, joint, and surgical sites. Using the antimicrobial susceptibility test, $75 \%$ of the tested isolates were found to be resistant to cefoxitin, $60 \%$ of isolates to ciprofloxacin, and $45 \%$ to tetracycline. Furthermore, resistance to oxacillin was $55 \%$, gentamicin $50 \%$, sulfamethoxazole/trimethoprim $47.5 \%$, clindamycin $47.5 \%$, levofloxacin $32.5 \%$ and rifampin $27.5 \%$. More than one third (37.5\%) of the isolates were multidrug-resistant.

By GelCompar II analysis, a total of 34 different pulsotypes were identified, including 3 clones (A, B, C) and 31 singletons. In each clone, 3 strains were found (Figure 1). Details of the strains are illustrated in Table 1.

Table 1. S. epidermidis Clones in Detail, Identified in the Al-Zahra Referral Hospital in Isfahan

\begin{tabular}{l|c|c|c}
\hline Clone Name & Strain & Ward & Specimen \\
\hline \multirow{4}{*}{ A } & A1 & Surgery & Ear wound \\
\cline { 2 - 4 } & A2 & NICU & Bloodstream \\
\cline { 2 - 4 } & A3 & NICU & Bloodstream \\
\hline \multirow{4}{*}{ B } & B1 & NICU & Bloodstream \\
\cline { 2 - 4 } & B1 & NICU & Bloodstream \\
\cline { 2 - 4 } & B2 & Oncology & Bloodstream \\
\hline \multirow{4}{*}{ C } & C1 & CCU & Catheter \\
\cline { 2 - 4 } & C1 & NICU & Eye wound \\
\cline { 2 - 4 } & C1 & ICU & Bloodstream \\
\hline
\end{tabular}

In total, 5 different STs were identified (ST2, ST5, ST27, ST29, and ST66). The 3 strains of clone A (A1, A2, and A3) belonged to ST2, ST27, and ST66; clone B (B1, B1 and B2) belonged to ST2, ST2, and ST20, whereas, the same 3 strains of clone C (C1) were related to ST5.

\section{Discussion}

The importance of S. epidermidis and related infections among coagulase negative staphylococci has been revealed in many studies (18). Villari et al. have described that $29.8 \%$ of surface infections and $39.8 \%$ of bloodstream infections belonged to S. epidermidis (19). Recently, as a result of the emergence of multidrug-resistant strains (MDR), the S. epidermidis nosocomial infections have become a major challenge in health care units. In our research, $37.5 \%$ of the isolates were determined as MDR with resistance to 3 different classes of antibiotics. In addition, a high rate of resistance to oxacillin (55\%) was found in our clinical isolates. Moreover, the majority of the strains were resistant to ciprofloxacin, tetracycline, and trimethoprim/sulfamethoxazole as well as oxacillin. These findings are consistent with other studies (20). Discussions regarding CoNS in blood cultures and hospital infections in numerous European and Asian countries have demonstrated that resistance to aminoglycosides and trimethoprim/sulfamethoxazole has increased in past two decades $(20,21)$. Increase of MDR strains and the importance of functional molecular typing methods in comparison to conventional techniques has motivated us to conduct the present study. This study, as the first report of S. epidermidis molecular epidemiology from a main tertiary referral hospital in Isfahan, Iran, characterized the 40 true clinical S. epidermidis isolates, using PFGE as a short-term epidemiological method. Our results showed that a total 34 different pulsotypes with 3 clones (A, B, and C) were distinguished. These results indicate that $S$. epidermidis has a high degree of genotypic diversity, as found in other investigations. For instance, in 2002, Raimundo et al. identified 43 subtypes in 55 isolates in a neonatal intensive care unit, although related studies have shown different clinical outcomes (22). However, we have found that a number of isolates were different in pulsotypes while, displaying a similar antibiotic resistance profile (strains 9 and 10 in this study), the association between the PFGE subtypes and various resistance profiles were equally observed as in the study of Miragaia et al. in 2002 (23). The microorganism gene exchange and common genetic events such as horizontal gene transfer and dissemination of mobile genetic elements may play an important role in pattern diversity (24).

As a result of $S$. epidermidis infections in different health care units, application of molecular epidemiology typing methods is a helpful technique for tracking the clones and controlling the further transmission of 


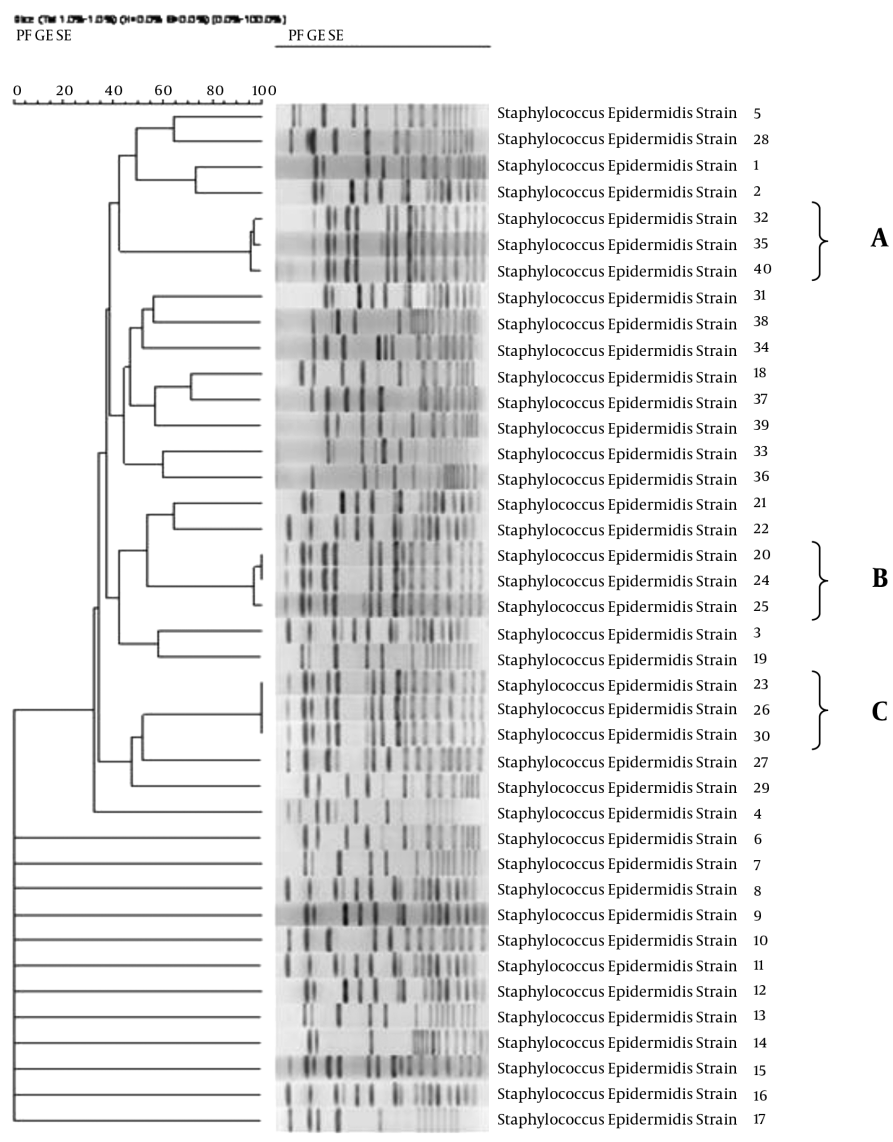

Figure 1. Pulsed-Field Gel Electrophoresis (PFGE) Dendrogram of 40 S. epidermidis Isolates Recovered from Al-Zahra Hospital, with a 90\% Similarity Cut-Off Point Clustered by UPGMA

infections. Amongst several methods for molecular typing, pulsed-field gel electrophoresis (PFGE) is the preferential method for $S$. epidermidis epidemiological studies and outbreaks (16). Furthermore, multilocus sequence typing (MLST) is known as an alternative molecular typing method for evaluating the taxonomic classification and microorganism genetic diversity in long-term epidemiological studies (17).

In the present study, the molecular characteristics of 3 clones (9 strains) in PFGE were examined using the MLST method. The MLST resulted in 5 distinct STs and showed that both ST2 and ST5 were the most represented sequence types. Although these STs have previously been detected in different studies, this is the first report originating from our tertiary referral hospital in Isfahan. Most studies have shown that ST2 is associated with positive isolates of the intercellular adhesion gene (ica), which has been disseminated in many regions and has caused a variety of serious infections. Comparatively, ST27 is highly adapted to hospi- tal environments since it is rarely found outside of medical facilities and can therefore be colonized in hospitalized patients in a short period of time $(25,26)$.

Considering the main clones, pulsotypes, and identified STs, the dissemination of MDR clones occurred in the hospital setting. These strains were found in different wards of the hospital such as the surgery and oncology wards, the neonatal intensive care unit (NICU), the intensive care unit (ICU), and the coronary care unit (CCU). This issue is more evidently seen in clone $C$, in which the first strain was isolated from the CCU ward from a catheter sample, the second strain from the NICU (eye wound) and the third from the ICU (bloodstream) (Table 1).

In accordance with the life cycle of S. epidermidis, both our results and the increase of MDR as well as invasive strains implicated in severe diseases, infection control procedures and prevention of colonization are essential for control of the epidemic strains in health care units. 


\section{Acknowledgments}

This research was supported by Isfahan University of Medical Sciences (grant no. 392003).

\section{Footnote}

Authors' Contribution: Study concept and design: Amirmorteza Ebrahimzadeh Namvar and Seyed Asghar Havaei. Analysis, interpretation of data, and preparation of the draft of the manuscript: Masoumeh Douraghi and Amirmorteza Ebrahimzadeh Namvar.

\section{References}

1. Heilmann C, Peters G. In: Gram-positive pathogens. Fischetti VA, Novick RP, Ferretti JJ, Portnoy DA, Rood JI, editors. Washington, D.C: American Society for Microbiology; 2000. pp. 442-8.Biology and pathogenesis of Staphylococcus epidermidis.

2. Namvar AE, Bastarahang S, Abbasi N, Ghehi GS, Farhadbakhtiarian $S$, Arezi P, et al. Clinical characteristics of Staphylococcus epidermidis: a systematic review. GMS Hyg Infect Control. 2014;9(3):Doc23. doi: 10.3205/dgkh000243. [PubMed: 25285267].

3. Warren JW. Catheter-associated urinary tract infections. Int J Antimicrob Agents. 2001;17(4):299-303. [PubMed: 11295412].

4. Havaei SA, Ebrahimzadeh Namvar A, Moghim S, Rastegar Lari A, Akbari M. Phenotypic and genotypic detection of Staphylococcus epidermidis delta toxin from neonatal patients in Isfahan and Tehran University hospitals. Mol Genetics Microbiol Virol. 2014;29(3):139-43. doi: 10.3103/s0891416814030070.

5. Bjorkqvist M, Soderquist B, Tornqvist E, Sjoberg L, Fredlund H, Kuhn I, et al. Phenotypic and genotypic characterisation of blood isolates of coagulase-negative staphylococci in the newborn. APMIS. 2002;110(4):332-9. [PubMed: 12076269].

6. Ziebuhr W, Hennig S, Eckart M, Kranzler H, Batzilla C, Kozitskaya S. Nosocomial infections by Staphylococcus epidermidis: how a commensal bacterium turns into a pathogen. Int J Antimicrob Agents. 2006;28 Suppl 1:S14-20. doi: 10.1016/j.ijantimicag.2006.05.012. [PubMed: 16829054].

7. Fitzpatrick F, Humphreys H, O'Gara JP. The genetics of staphylococcal biofilm formation-will a greater understanding of pathogenesis lead to better management of device-related infection? Clin Microbiol Infect. 2005;11(12):967-73. doi: 10.1111/j.1469-0691.2005.01274.x. [PubMed: 16307550].

8. Tan TQ, Musser JM, Shulman RJ, Mason EJ, Mahoney DJ, Kaplan SL. Molecular epidemiology of coagulase-negative Staphylococcus blood isolates from neonates with persistent bacteremia and children with central venous catheter infections. J Infect Dis. 1994;169(6):1393-7. [PubMed: 8195625].

9. Smith AJ, Robertson D, Tang MK, Jackson MS, MacKenzie D, Bagg J. Staphylococcus aureus in the oral cavity: a three-year retrospective analysis of clinical laboratory data. Br Dent J. 2003;195(12):701-3. doi: 10.1038/sj.bdj.4810832. [PubMed: 14718964] discussion 694.

10. Bogado I, Limansky A, Sutich E, Marchiaro P, Marzi M, Putero J, et al. Molecular characterization of methicillin-resistant coagulasenegative staphylococci from a neonatal intensive care unit. Infect Control Hosp Epidemiol. 2002;23(8):447-51. doi: 10.1086/502083. [PubMed: 12186210].

11. Garza-Gonzalez E, Lopez D, Pezina C, Muruet W, Bocanegra-Garcia V, Munoz I, et al. Diversity of staphylococcal cassette chromosome mec structures in coagulase-negative staphylococci and relationship to drug resistance. J Med Microbiol. 2010;59(Pt 3):323-9. doi: 10.1099/jmm.0.015800-0. [PubMed: 20007762].
12. Wielders CL, Vriens MR, Brisse S, de Graaf-Miltenburg LA, Troelstra A, Fleer A, et al. In-vivo transfer of mecA DNA to Staphylococcus aureus [corrected]. Lancet. 2001;357(9269):1674-5. [PubMed: 11425376].

13. National Nosocomial Infections Surveillance S. National Nosocomial Infections Surveillance (NNIS) System Report, data summary from January 1992 through June 2004, issued October 2004. Am JInfect Control. 2004;32(8):470-85. doi: 10.1016/S0196655304005425. [PubMed: 15573054].

14. CLSI . Performance standards for antimicrobial susceptibility testing; 21st informational supplement. M100-21. Wayne: CLSI; 2013.

15. Chung M, de Lencastre H, Matthews P, Tomasz A, Adamsson I, Aires de Sousa M, et al. Molecular typing of methicillin-resistant Staphylococcus aureus by pulsed-field gel electrophoresis: comparison of results obtained in a multilaboratory effort using identical protocols and MRSA strains. Microb Drug Resist. 2000;6(3):189-98. doi: 10.1089/mdr.2000.6.189. [PubMed: 11144419].

16. Tenover FC, Arbeit RD, Goering RV, Mickelsen PA, Murray BE, Persing $\mathrm{DH}$, et al. Interpreting chromosomal DNA restriction patterns produced by pulsed-field gel electrophoresis: criteria for bacterial strain typing. J Clin Microbiol. 1995;33(9):2233-9. [PubMed: 7494007].

17. Thomas JC, Vargas MR, Miragaia M, Peacock SJ, Archer GL, Enright MC. Improved multilocus sequence typing scheme for Staphylococcus epidermidis.J Clin Microbiol. 2007;45(2):616-9. doi: 10.1128/JCM.0193406. [PubMed: 17151213].

18. Akpaka PE, Christian N, Bodonaik NC, Smikle MF. Epidemiology of coagulase-negative Staphylococci isolated from clinical blood specimens at the University Hospital of the West Indies. West Indian Med J. 2006;55(3):170-3. [PubMed: 17087101].

19. Villari P, Sarnataro C, Iacuzio L. Molecular epidemiology of Staphylococcus epidermidis in a neonatal intensive care unit over a three-year period.J Clin Microbiol. 2000;38(5):1740-6. [PubMed: 10790091].

20. Koksal F, Yasar H, Samasti M. Antibiotic resistance patterns of coagulase-negative staphylococcus strains isolated from blood cultures of septicemic patients in Turkey. Microbiol Res. 2009;164(4):404-10. doi: 10.1016/j.micres.2007.03.004. [PubMed: 17475456].

21. Santos Sanches I, Mato R, de Lencastre H, Tomasz A, Cem Net Collaborators, the International C. Patterns of multidrug resistance among methicillin-resistant hospital isolates of coagulasepositive and coagulase-negative staphylococci collected in the international multicenter study RESIST in 1997 and 1998. Microb Drug Resist. 2000;6(3):199-211. doi: 10.1089/mdr.2000.6.199. [PubMed: 11144420].

22. Raimundo O, Heussler H, Bruhn JB, Suntrarachun S, Kelly N, Deighton MA, et al. Molecular epidemiology of coagulase-negative staphylococcal bacteraemia in a newborn intensive care unit. J Hosp Infect. 2002;51(1):33-42. doi: 10.1053/jhin.2002.1203. [PubMed: 12009818].

23. Miragaia M, Couto I, Pereira SF, Kristinsson KG, Westh H, Jarlov JO, et al. Molecular characterization of methicillin-resistantStaphylococcus epidermidis clones: evidence of geographic dissemination.J Clin Microbiol. 2002;40(2):430-8. [PubMed: 11825953].

24. Cerdeno-Tarraga A, Thomson N, Holden M, Sebaihia M, Parkhill J. Lost and found. Trends Microbiol. 2001;9(11):526-7. [PubMed: 11825692].

25. Cherifi S, Byl B, Deplano A, Nonhoff C, Denis O, Hallin M. Comparative epidemiology of Staphylococcus epidermidis isolates from patients with catheter-related bacteremia and from healthy volunteers. J Clin Microbiol. 2013;51(5):1541-7. doi: 10.1128/JCM.03378-12. [PubMed: 23486718].

26. Rohde H, Kalitzky M, Kroger N, Scherpe S, Horstkotte MA, Knobloch $\mathrm{JK}$, et al. Detection of virulence-associated genes not useful for discriminating between invasive and commensal Staphylococcus epidermidis strains from a bone marrow transplant unit.J Clin Microbiol. 2004;42(12):5614-9. doi: 10.1128/JCM.42.12.5614-5619.2004. [PubMed: 15583290]. 